\title{
Can abnormalities of ventricular repolarisation identify insulin dependent diabetic patients at risk of sudden cardiac death?
}

\author{
P J Weston, J M Glancy, P G McNally, H Thurston, D P de Bono
}

\begin{abstract}
Objective-To study the possible association of QT dispersion and mean QTc intervals, as measured from standard 12 lead electrocardiograms, with baroreceptor-cardiac reflex sensitivity (BRS) in insulin dependent diabetic patients. Design-Comparative study of noninvasive assessment of BRS, QT interval, and QT dispersion.

Setting-Large teaching hospital.

Subjects-31 young asymptomatic, normotensive, insulin dependent diabetic patients, aged 20-55 years with normal clinical autonomic function.

Methods-QT intervals and QT dispersion were measured by a single observer blinded to other data about the patients. BRS was measured after activating the baroreflex with a Valsalva manoeuvre, and the rate in change of $R-R$ interval to increasing systolic pressure during phase 4 was measured; in addition sequence analysis of resting systolic blood pressure and heart rate was performed during standing. The $\alpha$ coefficient-an index of the overall gain of the baroreflex mechanisms-was estimated from spectral analysis data of systolic blood pressure and pulse interval variability.
\end{abstract}

Results-Mean (SD) QTc interval was 406 (23) ms, QT dispersion was 44 (13) ms. There was no association between QT dispersion and any measurement of BRS. There was a negative correlation between mean QTc intervals and sequence analysis BRS $(r=-0.355, P=0.049)$, but no association with Valsalva BRS. The $\alpha$ coefficient, showed a significant negative correlation with mean QTc $(r=-0.42$, $\mathbf{P}=0.008$ )

Conclusions-Abnormal BRS may be reflected in the heart by global prolongation of ventricular repolarisation, but not by dispersion of ventricular repolarisation. This may, in part, explain the increase in sudden cardiac death seen in IDDM patients.

(Heart 1997;78:56-60)

Keywords: QT interval; QT dispersion; insulin dependent diabetes mellitus; baroreceptor-cardiac reflex sensitivity
There is increasing evidence for the role of the autonomic nervous system in the pathogenesis of sudden cardiac death resulting from ventricular arrhythmias. ${ }^{1}$ Sudden death in young insulin dependent diabetics is usually attributed to hypoglycaemia, often with little clinical evidence. ${ }^{2}$ In these patients the role of the autonomic nervous system may be important, although there is usually no clinical evidence of autonomic dysfunction. ${ }^{2}$ Several more sensitive measures of autonomic function have been proposed, which may prove useful in identifying patients at risk of sudden death. Baroreceptor-cardiac reflex sensitivity (BRS), assessed from the linear relation between systolic blood pressure and $\mathbf{R}-\mathbf{R}$ interval, has recently been applied to the study of autonomic function in diabetic patients. ${ }^{34}$ Using spectral analysis methods to study heart rate and blood pressure variability allows quantification of relative vagal and sympathetic influences on the heart. ${ }^{56}$ The power spectra of heart rate and systolic blood pressure variability has been shown to consist of three major peaks-very low, low, and high frequency. Heart rate fluctuations in the low frequency band $(0.05-0.15 \mathrm{~Hz})$ are said to represent predominately sympathetic function with a contribution from the parasympathetic system, while the high frequency peak $(0 \cdot 2-0.35$ $\mathrm{Hz}$ ) is said to mainly reflect vagal, or parasympathetic, activity. ${ }^{5}$ Using spectral analysis methods the ratio of low frequency to high frequency power can be taken as a measure of sympathovagal balance. ${ }^{7}$ Furthermore, the average gain of the transfer function between $\mathbf{R}-\mathbf{R}$ interval and systolic blood pressure variability in both low and high frequency bandwidths acts as an assessment of baroreceptor-cardiac reflex sensitivity and is usually defined as the $\alpha$ coefficient. ${ }^{8}$ It is thus possible to identify abnormalities of autonomic regulation of heart rate and systolic blood pressure. Such abnormalities may be expressed in the heart as abnormalities of cardiac repolarisation. Prolongation of the QT interval has been recognised in diabetic patients with autonomic dysfunction. ${ }^{9} 10$ The interlead variability of QT intervals-the QT dispersion-may provide more detailed information about dispersion of cardiac repolarisation, and has been suggested as a measure of arrhythmic risk in a number of settings. ${ }^{11} \mathrm{We}$ measured the association between sympathovagal balance and BRS with $Q T$ intervals and 
QT dispersion in 31 healthy insulin dependent diabetic patients.

\begin{abstract}
Methods
SUBJECTS

Thirty one insulin dependent diabetic patients (20 males) aged between 20 and 54 years, mean 34.8 (SD 5.4), were studied. Patients were recruited from the diabetic clinic at Leicester Royal Infirmary and all gave informed consent. All patients had a history of ketonuria at the time of diagnosis. Patients with evidence of microvascular disease (that is, those with microalbuminuria, retinopathy, or clinical evidence of neuropathy) were excluded, as were those with clinical evidence of peripheral or cardiac vascular disease or a history of cerebrovascular disease. Patients were all normotensive, with clinic blood pressure of $<140 / 90 \mathrm{~mm} \mathrm{Hg}$ on at least three occasions and were not taking medication other than insulin.
\end{abstract}

\section{METHODS}

Subjects attended the test laboratory at least two hours postprandially, and the tests were performed in a quiet room with the temperature controlled at $20-24^{\circ} \mathrm{C}$. Subjects were part of a larger study looking at autonomic function in patients with insulin dependent diabetes mellitus. No subjects smoked and all abstained from caffeine for at least 12 hours before the test day. All subjects had height and weight measured, from which body mass index was calculated, and waist:hip ratio was also measured. All subjects performed five standard cardiovascular tests of autonomic function. ${ }^{1}$

After resting for 10 minutes, the Finapres device - which records beat to beat blood pressure and has been validated against intra-arterial measurements in a variety of clinical settings including during the Valsalva manoeuvre ${ }^{12}-$ was applied to the middle finger of the left hand and a surface ECG fitted to record R-R interval. Subjects were initially supine, with the arm supported at the level of the right atrium, and patients were asked not to talk. The Finapres was calibrated and when stable, resting systolic blood pressure and $\mathbf{R}-\mathbf{R}$ interval recorded for 15 minutes. During this time the self-servo mechanism of the Finapres was disabled. The subjects were then asked to stand, again with the arm supported at the level of the right atrium, and after a five minute resting period, during which the Finapres was recalibrated, the systolic blood pressure and R-R interval were recorded for a further 15 minutes. The respiratory rate was monitored throughout the recording periods and the recording was discontinued if the respiratory rate fell below 15 breaths/min. Data were converted from analogue to digital using an on-line personal computer sampling at $200 \mathrm{~Hz}$. A QRS detection algorithm was employed to automatically mark the $\mathbf{R}-\mathbf{R}$ intervals (from the surface ECG). The computer software also detected increases and decreases in systolic blood pressure and compared these to changes in $R-R$ interval. Where three or more consecutive systolic blood pressure readings increased by $\geqslant 0.5 \mathrm{~mm} \mathrm{Hg} / \mathrm{s}$, pulse inter- val was automatically compared and if $R-R$ interval lengthened this was counted as an "up BRS" sequence. Similarly, where systolic blood pressure decreased for three or more consecutive readings by $\geqslant 0.5 \mathrm{~mm} \mathrm{Hg} / \mathrm{s}$, and this was associated with a decrease in $\mathrm{R}-\mathrm{R}$ interval this was counted as a "down BRS" sequence. BRS was determined from the regression of $R-R$ interval on systolic blood pressure for all up, down, and non-BRS sequences (that is, where changes in systolic blood pressure were not associated with changes in R-R interval). Only regression lines with a correlation coefficient of $\geqslant 0.8$ and significant at $P<0.05$ were used, and an average BRS for all of the up and down BRS sequences was calculated. Furthermore, using the same data, power spectra were estimated using a fast Fourier transformation of supine pulse and blood pressure data. This analysis allows calculation of power in two frequency bands: the low frequency band $(0.05-0.15 \mathrm{~Hz})$ and the high frequency band $(0.2-0.35 \mathrm{~Hz})$. The power of the low frequency is said to represent sympathetic activity and the high frequency represents vagal activity. ${ }^{5}$ Finally, subjects were asked to perform three Valsalva manoeuvres. The blood pressure overshoot after release of the strain (phase 4) results in a baroreflex mediated slowing of heart rate and BRS was estimated from the regression of R-R interval on systolic blood pressure. ${ }^{413}$ The Valsalva manoeuvre was explained to the subjects, who then blew a forced expiration of $\mathbf{4 0}$ $\mathrm{mm} \mathrm{Hg}$ for 15 seconds. BRS was calculated as the average of the three Valsalva manoeuvres. The tests were performed by one investigator and analysed by the same person.

All patients had a 12 lead simultaneous ECG (Hewlett Packard Pagewriter) recorded at rest. Each ECG had at least 10 leads in which a QT interval could be accurately measured. A single observer, blinded to the other data, analysed all recordings.

Electrocardiograms were scanned by a flatbed scanner interfaced with a personal computer. Each image was then cut and copied into 12 files, corresponding to the 12 leads of the electrocardiogram. Specially designed software skeletonised and joined each image. Each image can be magnified on the computer monitor for optimal QT interval measurement using a mouse. This method has been validated previously. ${ }^{14}$ QT interval measurements were by standard criteria. Leads were excluded from QT dispersion analysis if the $T$ wave, or its terminal portion, were too flat to determine the $T$ wave end accurately, or if a $U$ wave so interfered with a $T$ wave end that the end of the $T$ wave could not be identified accurately. Maximum and mean QT intervals for all 12 leads were rate corrected using Bazett's formula. QT dispersion is the difference between the longest and shortest non-rate-corrected QT interval across the surface electrocardiogram.

\section{STATISTICS}

Results are expressed as mean (SD). After confirming normality of data with ShapiroWilks' $W$ test, data were compared by Pearson's correlation coefficient and least 
Table 1 Measures of autonomic function, $Q T c$ and $Q T$ dispersion. Values are mean (SD)

\begin{tabular}{|c|c|c|}
\hline 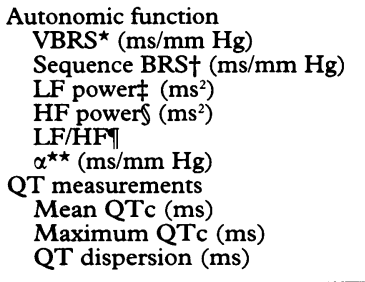 & \multicolumn{2}{|c|}{$\begin{array}{c}3 \cdot 2(1 \cdot 4) \\
5 \cdot 1(2 \cdot 7) \\
582 \cdot 9(36 \cdot 1) \\
77 \cdot 9(20 \cdot 2) \\
8 \cdot 8(2 \cdot 7) \\
5 \cdot 3(2 \cdot 1) \\
\\
406(22) \\
428(22) \\
44(13)\end{array}$} \\
\hline \multicolumn{3}{|c|}{$\begin{array}{l}\text { *alsalva derived baroreceptor-cardiac reflex sensitivity } \\
\text { tstanding sequence analysis derived baroreceptor-cardiac } \\
\text { reflex sensitivity; ftotal power of low frequency band } \\
(0.05-0.15 \mathrm{~Hz}) ; \text { jtotal power of high frequency band } \\
(0.2-0.35 \mathrm{~Hz}) ; \text { ๆratio of low frequency to high frequency } \\
\text { power; } \star \star \alpha \text { values (mean baroreceptor-cardiac reflex sensitivity } \\
\text { for low and high frequency bands). }\end{array}$} \\
\hline \multicolumn{3}{|c|}{$\begin{array}{l}\text { Table } 2 \text { Correlation of mean } Q T c \text { intervals with } \\
\text { autonomic function measurements }\end{array}$} \\
\hline & & $P$ \\
\hline 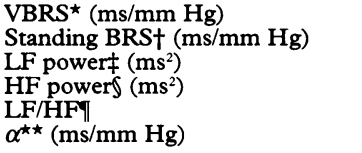 & $\begin{array}{l}-0.02 \\
-0.36 \\
-0.42 \\
-0.42 \\
-0.5 \\
-0.42\end{array}$ & $\begin{array}{l}0.9 \\
0.05 \\
0.02 \\
0.02 \\
0.01 \\
0.008\end{array}$ \\
\hline
\end{tabular}

*Valsalva derived baroreceptor-cardiac reflex sensitivity; tstanding sequence analysis derived baroreceptor-cardiac reflex sensitivity; łtotal power of low frequency band $0.05-0.15 \mathrm{~Hz}$ ); dtotal power of high frequency band $(0.2-0.35 \mathrm{~Hz})$; fratio of low frequency to high frequency power; ${ }^{\star \star}$ mean baroreceptor-cardiac reflex sensitivity for low
and high frequency bands.

squares regression analysis. The determinants of QTc and QT dispersion were assessed using multiple regression analysis with age, systolic blood pressure, duration of diabetes, and BRS as the independent predictor variables. A $\mathbf{P}$ value of $<0.05$ was regarded as statistically significant.

\section{Results}

Mean and standard deviation of measures of autonomic function, QTc intervals, and QT dispersion are presented in table 1 . Table 2 shows the correlation between measures of autonomic function and mean QTc intervals.

There were significant negative correlations between mean QTc intervals and standing sequence derived BRS (fig 1). Furthermore, the $\alpha$ coefficient derived from spectral studies (fig 2) and the ratio of low frequency to high frequency power showed significant negative

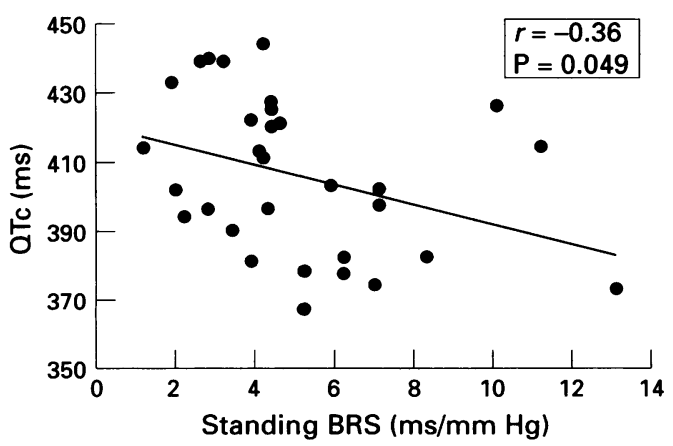

Figure 1 Relation between mean $Q T c$ and sequence derived baroreceptor-cardiac reflex sensitivity (standing $B R S)$.

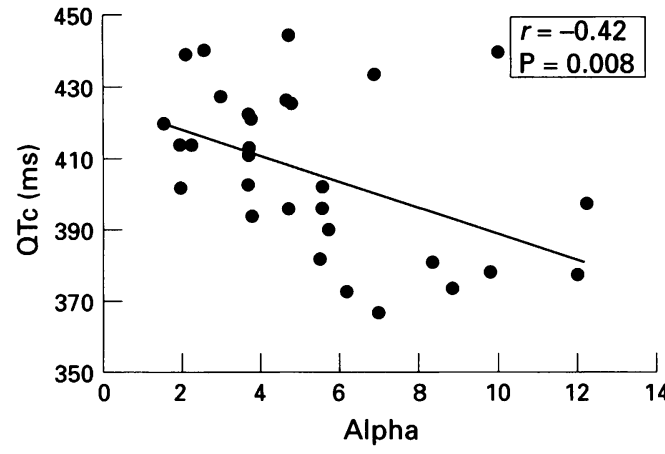

Figure 2 Relation between mean $Q T c$ and

baroreceptor-cardiac reflex sensitivity derived from spectral analysis data of $R-R$ interval and systolic blood pressure variability ( $\alpha$ coefficient).

correlations with mean QTc that were independent of age, systolic blood pressure, and duration of diabetes. There were no associations between either maximum QTc intervals or QT dispersion and any measure of autonomic function.

\section{Discussion}

This study suggests a link between global measurement of cardiac repolarisation, as expressed by prolonged mean QTc intervals, and measures of autonomic function. In contrast dispersion of cardiac repolarisation-suggested as an important mechanism in the development of cardiac arrhythmias-does not appear to be associated with the tests of autonomic function studied.

There is a well described association between abnormalities of autonomic function and sudden death in diabetics. ${ }^{1}$ Moreover prolonged QT intervals may be associated with mortality in diabetic patients. ${ }^{9}$ It is possible that abnormalities in autonomic function induced by diabetes are expressed by prolongation of cardiac repolarisation, and that the changes in QT intervals are the pathway for arrhythmia generation and sudden death in these patients. Certainly patients with clinical evidence of diabetic neuropathy do have abnormal QT intervals though more subtle abnormalities of autonomic function have not previously been examined in any detail. ${ }^{9} 1015$

There is considerable evidence that the QT interval on the surface ECG is to some extent determined by the autonomic nervous system. ${ }^{16}$ Manipulation of the autonomic nervous system by procedures such as left stellate ganglionectomy produces QT interval prolongation. ${ }^{17}$ Moreover a direct vagal activity on the QT interval has also been demonstrated. ${ }^{18}$ More specifically in diabetic patients with clinical autonomic neuropathy, QT interval prolongation has been shown, and it has been proposed that it may have a role in sudden cardiac death in these patients. ${ }^{915}$ One study has suggested that diabetic neuropathy is associated with a decrease in QTc intervals ${ }^{19}$; however, there was a difference in the heart rates of the groups studied which may have lead to overcorrection of QT intervals by Bazett's formula. The data presented are in agreement 
with the majority of evidence that abnormalities of autonomic function in diabetic patients are reflected in global prolongation of cardiac repolarisation and prolonged QT intervals. In a study by Wei and colleagues, looking at diabetic patients with abnormal bedside tests of autonomic function, ${ }^{20}$ QT dispersion was increased compared to a smaller group of nondiabetic subjects. The patients we studied all had clinically normal autonomic function; therefore it seems likely that the more subtle abnormalities of autonomic function demonstrated in our apparently healthy diabetic patients might also be expressed in globally prolonged cardiac repolarisation.

Few data are available about the role of the autonomic nervous system in dispersion of the QT interval. These data suggest that the autonomic nervous system is not important in determining dispersion of cardiac repolarisation, and therefore in QT dispersion measurement. One recent study has described QT dispersion in patients with primary autonomic failure, showing that it was no different between the patients studied and controls, but interestingly that QT and QTc intervals were prolonged in patients who had primary autonomic failure. ${ }^{21}$ In contrast, in a study of 13 patients with diabetic nephropathy it has been suggested that severe autonomic neuropathy is associated with increased QT dispersion. ${ }^{22}$ However, these patients also had a high incidence of coronary artery disease, and other cardiac problems, which could have been responsible for the observed increase in QT dispersion. It can thus be postulated that abnormalities of autonomic function are reflected in prolongation of the QT interval, whereas QT dispersion abnormalities may only develop as a result of myocardial damage.

Spectral analysis methods of analysing resting heart rate and systolic blood pressure variations are increasingly used to study diabetic autonomic neuropathy. ${ }^{56}$ Studies in diabetic subjects with autonomic neuropathy have shown that there is a marked loss of power in the high frequency region, suggesting an abnormality of parasympathetic function and thus a relative increase in sympathovagal balance. ${ }^{61323}$

In the present study we found significant negative correlations between measures of autonomic function and mean QT interval. There was a further significant negative correlation between baroreceptor gain $(\alpha)$ and QTC which we assume to be a reflection of autonomic dysfunction, and more specifically parasympathetic function, rather than a structural abnormality of the baroreceptor itself.

Previous studies looking at the circadian rhythm of sympathovagal balance have found an increase in sympathetic activity in the morning and a relative increase in parasympathetic tone during the night. ${ }^{7}$ There is reversal of the normal pattern in diabetic subjects, namely a decrease of vagal activity during the night and thus a relative sympathetic predominance. An increase in cardiovascular events during night time has also been reported in patients with insulin dependent diabetes, rais- ing the possibility that a relative sympathetic overactivity may in part be responsible. ${ }^{24}$ It is interesting to postulate that in those diabetic patients with an already prolonged QTc, a relative sympathetic predominance makes ventricular arrhythmias more likely and could explain some of the "dead in bed" cases. One study has found an increase in QTc during experimentally induced hypoglycaemia ${ }^{25}$ and it is conceivable that nocturnal hypoglycaemia further prolongs the QTc and increases the risk of developing ventricular arrhythmia.

We found no significant relations between QT dispersion or QTc and BRS derived from the Valsalva manoeuvre. The Valsalva manoeuvre is difficult to perform and relies on an intact baroreceptor mechanism as well as parasympathetic and sympathetic function. The patients studied were all asymptomatic with no clinical evidence of autonomic dysfunction, so subtle abnormalities of sympathovagal balance may go undetected in such a crude test.

In conclusion these data suggest that abnormal baroreceptor-cardiac reflex sensitivity, due in part to impaired parasympathetic function, may be reflected in the heart by global prolongation of ventricular repolarisation, but not by dispersion of ventricular repolarisation. Such abnormalities may play some part in the sudden cardiac deaths seen in young insulin dependent diabetic patients.

1 Ewing DJ, Martyn CN, Young RJ, Clarke BF. The value of cardiovascular autonomic function tests: a ten year experience in diabetes. Diabetes Care 1985;8:491-8.

2 Tattersall RB, Gill GV. Unexplained sudden death of type 1 diabetic patients. Diabetic Med 199;8:49-58.

3 Bristow A, Honour AJ, Pickering GW, Sleight P, Smith HS. Diminished baroreflex sensitivity in high blood pressure. Circulation 1969;39:48-54.

4 Ferrer T, Kennedy WR, Sahinen F. Baroreflexes in patients with diabetes mellitus. Neurology 1991;41:1462-6.

5 Akselrod S, Gordon D, Ubel FA, Shannon DC, Barger AC, Cohen RJ. Power spectrum analysis of the heart rate fluctuation: a quantitative probe of beat-to-beat cardiofluctuation: a quantitative probe of beat-
vascular control. Science 1981;213:220-2

6 Lishner M, Akselrod S, Mor Avi V, Oz O, Divon M, Ravid $M$. Spectral analysis of heart rate fluctuations. A noninvasive sensitive method for the early diagnosis of autonomic neuropathy in diabetes mellitus. $\mathcal{F}$ Auton Nerv Syst 1987;19:119-25.

7 Bernardi L, Ricordi L, Lazzari P, Solda P, Calciati A, Ferrari MR, et al. Impaired circadian modulation of sympathovagal activity in diabetes. A possible explanation for altered temporal onset of cardiovascular disease. Circulation 1992;86:1443-52.

8 Lucini D, Pagani M, Mela S, Malliani A. Sympathetic restraint of baroreflex control of heart period in normotensive and hypertensive subjects. Clin Sci 1994;86: 547-56.

9 Bellavere F, Ferri M, Guarini L, Bax G, Piccoli A, Cardone $\mathrm{C}$, et al. Prolonged QT period in diabetic autonomic neuC, et al. Prolonged QT period in diabetic autonomic neuropathy: a possible

10 Veglio M, Chinaglia A, Borra M, Cavallo Perin P. Does abnormal QT interval prolongation reflect autonomic dysfunction in diabetic patients? QTc interval measure versus standardised tests in diabetic autonomic neuropathy. Diabetic Med 1995;12:302-6.

11 Higham PD, Campbell RWF. QT dispersion. Br Heart $\mathcal{f}$ 1994;71:508-10.

12 Omboni S, Parati G, Frattola A, Mutti E, Di Rienzo, Castiglioni $\mathrm{P}$, et al. Spectral and sequence analysis of finger blood pressure variability: comparison with analysis of intra-arterial recordings. Hypertension 1993;22:26-33.

13 Palmero HA, Caeiro TF, Iosa DJ, Bas J. Baroreceptor reflex sensitivity derived from phase 4 of the Valsalva maneuver. Hypertension 1981;3(suppl II):II134-7.

14 Bhullar HK, Fothergill JC, Goddard WP, De Bono D. Automated measurement of QT interval dispersion from hard-copy ECGs. 7 Electrocardiol 1993;26:321-31. 15 Ewing DJ, Neilson JMM. QT interval length and diabetic
autonomic neuropathy. Diabetic Med 1990;7:23-6.

16 Rosen MR, Jeck CD, Steinberg SF. Autonomic modulation of cellular repolarisation and of electrocardiographic 
17 Abildskov JA. Sympathetic imbalance hypothesis of QT interval prolongation. $\mathcal{F}$ Cardiovasc Electrophysiol 1991;2: 355-9.

18 Ahnve $S$, Vallin $H$. Influence of heart rate and inhibition of autonomic tone on the QT interval. Circulation 1982;65:435-9.

9 Ong JJC, Sarma JS, Venkataraman K, Levin SR, Singh BN. Circadian rhythmicity of heart rate and QTc interval in diabetic autonomic neuropathy: implications for the mechanisms of sudden death. Am Heart $\mathcal{F}$ 1993;125: 744-52.

20 Wei K, Dorian P, Newman D, Langer A. Association between QT dispersion and autonomic dysfunction in patients with diabetes mellitus. $\mathcal{F} \mathrm{Am} \mathrm{Col} \mathrm{Cardiol} \mathrm{1995;26:}$ 859-63.

21 Lo SS, Mathias CJ, St John Sutton M. QT interval and dis- persion in primary autonomic failure. Heart 1996;75: 498-501.

22 Kirvelä M, Yli-Hankala A, Lindgren L. QT dispersion and autonomic function in diabetic and non-diabetic patients with renal failure. $\operatorname{Br} \mathcal{F}$ Anaesth 1994;73:801-4.

23 Parati G, Saul P, Di Rienzo M, Mancia G. Spectral analysis of blood pressure and heart rate variability in evaluating cardiovascular regulation: a critical appraisal. Hypertension 1995;25:1276-86.

24 Fava S, Azzopardi J, Muscat H, Fenech FF. Absence of circadian variation in the onset of acute myocardial infarction in diabetic subjects. Br Heart $\mathcal{f} 1995 ; 74: 370-2$.

25 Heller SR, Marques JLB, George E, Harris ND, Cochrane T. QTc interval prolongation during hypoglycaemia in patients with IDDM - a possible mechanism of sudden death [abstract]. Diabetologia 1995;38(suppl 1):A18. 